Mathematical Foundations of Computer Science 2003, 28th International Symposium Proceedings: Branislav Rovan, Peter Vojtás (eds.)

Springer Lecture Notes in Computer Science 2747 (2003), 429-438.

\title{
Local LTL with past constants is expressively complete for Mazurkiewicz traces ${ }^{\star}$
}

\author{
Paul Gastin ${ }^{1}$, Madhavan Mukund ${ }^{2}$, and K. Narayan Kumar ${ }^{2}$ \\ ${ }^{1}$ LIAFA, Université Paris 7, 2, place Jussieu, F-75251 Paris Cedex 05 \\ Paul.Gastin@liafa.jussieu.fr \\ 2 Chennai Mathematical Institute, 92 G N Chetty Road, Chennai 600 017, India \\ madhavan, kumar\}@cmi.ac.in
}

\begin{abstract}
To obtain an expressively complete linear-time temporal logic (LTL) over Mazurkiewicz traces that is computationally tractable, we need to intepret formulas locally, at individual events in a trace, rather than globally, at configurations. Such local logics necessarily require past modalities, in contrast to the classical setting of LTL over sequences. Earlier attempts at defining expressively complete local logics have used very general past modalities as well as filters (side-conditions) that "look sideways" and talk of concurrent events. In this paper, we show that it is possible to use unfiltered future modalities in conjunction with past constants and still obtain a logic that is expressively complete over traces.

Keywords Temporal logics, Mazurkiewicz traces, concurrency
\end{abstract}

\section{Introduction}

Linear-time temporal logic (LTL) [17] has established itself as a useful formalism for specifying the interleaved behaviour of reactive systems. To combat the combinatorial blow-up involved in describing computations of concurrent systems in terms of interleavings, there has been a lot of interest in using temporal logic more directly on labelled partial orders.

Mazurkiewicz traces [13] are labelled partial orders generated by dependence alphabets of the form $(\Sigma, D)$, where $D$ is a dependence relation over $\Sigma$. If $(a, b) \notin$ $D, a$ and $b$ are deemed to be independent actions that may occur concurrently. Traces are a natural formalism for describing the behaviour of static networks of communicating finite-state agents [24].

LTL over $\Sigma$-labelled sequences is equivalent to $\mathrm{FO}_{\Sigma}(<)$, the first-order logic over $\Sigma$-labelled linear orders [12] and thus defines the class of aperiodic languages over $\Sigma$. Though $\mathrm{FO}_{\Sigma}(<)$ permits assertions about both the past and the future, future modalities suffice for establishing the expressive completeness of LTL with respect to $\mathrm{FO}_{\Sigma}(<)[8]$. From a practical point of view, a finite-state program may be checked against an LTL specification relatively efficiently.

\footnotetext{
* Partial support of CEFIPRA-IFCPAR Project 2102-1 (ACSMV) is gratefully acknowledged.
} 
The first expressively complete temporal logic over traces was described in [6] for finite traces and in [19] for infinite traces. The result was refined in [4] to show expressive completeness without past modalities, using an extension of the proof technique developed for LTL in [23]. Formulas in both these logics are defined at global configurations (maximal antichains). Unfortunately, reasoning at the level of global configurations makes the complexity of deciding satisfiability non-elementary [21]. Computational tractability seems to require interpreting formulas at local states - effectively at individual events.

Recently, in [10], a local temporal logic has been defined over traces and shown to be expressively complete and tractable (the satisfiability problem is in PSPACE). This logic uses both future and past modalities (similar to the until and since operators of LTL) which are further equipped with filters (side-conditions). It was also shown that for finite traces, a restricted form of past modalities suffices, but only in conjunction with filtered future modalities. Another proposal is presented in [1] and this logic also uses the since operator.

LTL without any past operators is expressively complete over words but this cannot be the case for traces: there exist two first-order inequivalent traces that cannot be distinguished using only future modalities [22].

In this paper, we show that a very limited ability to talk about the past is sufficient to obtain expressive completeness over traces. Our logic uses unfiltered future modalities and a finite number of past constants. (In particular, there is no nesting of past operators and for that matter even future formulas cannot be nested into past formulas.) As in $[3,4,10]$, we show expressive completeness using an extension to traces of the proof technique introduced in [23] for LTL over sequences. From the recent general result proved in [9], it follows that the satisfiability problem for this new logic is also in PSPACE.

The paper is organized as follows. We begin with some preliminaries about traces. In Section 3 we define our new temporal logic. Section 4 describes a syntactic partition of traces that is used in Section 5 to establish expressive completeness. Many proofs have had to be omitted in this extended abstract. A full version of the paper is available in [11].

\section{Preliminaries}

We briefly recall some notions about Mazurkiewicz traces (see [5] for background). A dependence alphabet is a pair $(\Sigma, D)$ where the alphabet $\Sigma$ is a finite set of actions and the dependence relation $D \subseteq \Sigma \times \Sigma$ is reflexive and symmetric. The independence relation $I$ is the complement of $D$. For $A \subseteq \Sigma$, the set of letters independent of $A$ is denoted by $I(A)=\{b \in \Sigma \mid(a, b) \in I$ for all $a \in A\}$ and the set of letters depending on (some action in) $A$ is denoted by $D(A)=\Sigma \backslash I(A)$.

A Mazurkiewicz trace is a labelled partial order $t=[V, \leq, \lambda]$ where $V$ is a set of vertices labelled by $\lambda: V \rightarrow \Sigma$ and $\leq$ is a partial order over $V$ satisfying the following conditions: For all $x \in V$, the downward set $\downarrow x=\{y \in V \mid y \leq x\}$ is finite, $(\lambda(x), \lambda(y)) \in D$ implies $x \leq y$ or $y \leq x$, and $x \lessdot y$ implies $(\lambda(x), \lambda(y)) \in D$, where $\lessdot=<\backslash<^{2}$ is the immediate successor relation in $t$. 
The alphabet of a trace $t$ is the set $\operatorname{alph}(t)=\lambda(V) \subseteq \Sigma$ and its alphabet at infinity, alphinf $(t)$, is the set of letters occurring infinitely often in $t$. The set of all traces is denoted by $\mathbb{R}(\Sigma, D)$ or simply by $\mathbb{R}$. A trace $t$ is called finite if $V$ is finite. For $t=[V, \leq, \lambda] \in \mathbb{R}$, we define $\min (t) \subseteq V$ as the set of all minimal vertices of $t$. We can also read $\min (t) \subseteq \Sigma$ as the set of labels of the minimal vertices of $t$. It will be clear from the context what we actually mean.

Let $t_{1}=\left[V_{1}, \leq_{1}, \lambda_{1}\right]$ and $t_{2}=\left[V_{2}, \leq_{2}, \lambda_{2}\right]$ be a pair of traces such that alphinf $\left(t_{1}\right) \times \operatorname{alph}\left(t_{2}\right) \subseteq I$. We then define the concatenation of $t_{1}$ and $t_{2}$ to be $t_{1} \cdot t_{2}=[V, \leq, \lambda]$ where $V=V_{1} \cup V_{2}$ (assuming wlog that $V_{1} \cap V_{2}=\emptyset$ ), $\lambda=\lambda_{1} \cup \lambda_{2}$ and $\leq$ is the transitive closure of the relation $\leq_{1} \cup \leq_{2} \cup\left(V_{1} \times V_{2} \cap \lambda^{-1}(D)\right)$. The set of finite traces is then a monoid, denoted $\mathbb{M}(\Sigma, D)$ or simply $\mathbb{M}$, with the empty trace $1=(\emptyset, \emptyset, \emptyset)$ as unit.

Here is some useful notation for subclasses of traces. For $C \subseteq \Sigma$, let $\mathbb{R}_{C}=$ $\{t \in \mathbb{R} \mid \operatorname{alph}(x) \subseteq C\}$ and $\mathbb{M}_{C}=\mathbb{M} \cap \mathbb{R}_{C}$. Also, (alph $\left.=C\right)=\{t \in \mathbb{R} \mid$ $\operatorname{alph}(t)=C\},(\operatorname{alphinf}=C)=\{t \in \mathbb{R} \mid \operatorname{alphinf}(t)=C\}$ and $(\min =C)=\{t \in$ $\mathbb{R} \mid \min (t)=C\}$. For $A, C \subseteq \Sigma$, we set $\mathbb{R}_{C}^{A}=\mathbb{R}_{C} \cap($ alphinf $=A)$. Observe that $\mathbb{M}_{C}=\mathbb{R}_{C}^{0}$.

The first order theory of traces $\mathrm{FO}_{\Sigma}(<)$ is given by the syntax:

$$
\varphi::=P_{a}(x)|x<y| \neg \varphi|\varphi \vee \varphi| \exists x \varphi,
$$

where $a \in \Sigma$ and $x, y \in$ Var are first order variables. Given a trace $t=[V, \leq, \lambda]$ and a valuation $\sigma$ : Var $\rightarrow V, t, \sigma \models \varphi$ denotes that $t$ satisfies $\varphi$ under $\sigma$. We interpret each predicate $P_{a}$ by the set $\{x \in V \mid \lambda(x)=a\}$ and the relation $<$ as the strict partial order relation of $t$. The semantics then lifts to all formulas as usual. Since the meaning of a closed formula (sentence) $\varphi$ is independent of the valuation $\sigma$, we can associate with each sentence $\varphi$ the language $\mathcal{L}(\varphi)=\{t \in \mathbb{R} \mid$ $t=\varphi\}$. We say that a trace language $L \subseteq \mathbb{R}$ is expressible in $\mathrm{FO}_{\Sigma}(<)$ if there exists a sentence $\varphi \in \mathrm{FO}_{\Sigma}(<)$ such that $L=\mathcal{L}(\varphi)$. We denote by $\mathrm{FO}_{(\Sigma, D)}(<)$ the set of trace languages $L \subseteq \mathbb{R}(\Sigma, D)$ that are expressible in $\mathrm{FO}_{\Sigma}(<)$. For $n>0, \mathrm{FO}_{\Sigma}^{n}(<)$ denotes the set of formulas with at most $n$ distinct variables (note that each variable may be bound and reused several times).

We use the algebraic notion of recognizability. Let $h: \mathbb{M} \rightarrow S$ be a morphism to a finite monoid $S$. For $t, u \in \mathbb{R}$, we say that $t$ and $u$ are $h$-similar, denoted $t \sim_{h} u$, if either $t, u \in \mathbb{M}$ and $h(t)=h(u)$ or $t$ and $u$ have infinite factorizations in non-empty finite traces $t=t_{1} t_{2} \cdots, u=u_{1} u_{2} \cdots$ with $h\left(t_{i}\right)=h\left(u_{i}\right)$ for all $i$. The transitive closure $\approx_{h}$ of $\sim_{h}$ is an equivalence relation. Since $S$ is finite, this equivalence relation is of finite index with at most $|S|^{2}+|S|$ equivalence classes. A trace language $L \subseteq \mathbb{R}$ is recognized by $h$ if it is saturated by $\approx_{h}$ (or equivalently by $\sim_{h}$ ), i.e., $t \in L$ implies $[t]_{\approx_{h}} \subseteq L$ for all $t \in \mathbb{R}$.

Let $L \subseteq \mathbb{R}$ be recognized by a morphism $h: \mathbb{M} \rightarrow S$. For $B \subseteq \Sigma, L \cap \mathbb{M}_{B}$ and $L \cap \mathbb{R}_{B}$ are recognized by $h \uparrow_{\mathbb{M}_{B}}$ the restriction of $h$ to $\mathbb{M}_{B}$. A finite monoid $S$ is aperiodic if there is an $n \geq 0$ such that $s^{n}=s^{n+1}$ for all $s \in S$. A trace language $L \subseteq \mathbb{R}$ is aperiodic if it is recognized by some morphism to a finite and aperiodic monoid. First-order definability coincides with aperiodicity for traces. 
Theorem $1([6,7])$. A language $L \subseteq \mathbb{R}(\Sigma, D)$ is expressible in $\mathrm{FO}_{\Sigma}(<)$ if and only if it is aperiodic.

\section{Local temporal logic}

We denote by LocTL $L_{\Sigma}^{\mathrm{i}}$ the set of (internal) formulas over the alphabet $\Sigma$. They are given by the following syntax:

$$
\varphi::=a \in \Sigma|\neg \varphi| \varphi \vee \varphi|\operatorname{EX} \varphi| \varphi \mathrm{U} \varphi \mid \neg a \mathrm{~S} b, a, b \in \Sigma
$$

Let $t=[V, \leq, \lambda] \in \mathbb{R}$ be a finite or infinite trace and let $x \in V$ be some vertex of $t$. We write $t, x \models \varphi$ to denote that trace $t$ at node $x$ satisfies the formula $\varphi \in \operatorname{LocTL}_{\Sigma}^{\mathrm{i}}$. This is defined inductively as follows:

$$
\begin{array}{ll}
t, x=a & \text { if } \lambda(x)=a \\
t, x=\neg \varphi & \text { if } t, x \not \models \varphi \\
t, x=\varphi \vee \psi & \text { if } t, x \models \varphi \text { or } t, x \models \psi \\
t, x=\operatorname{EX} \varphi & \text { if } \exists y . x \lessdot y \text { and } t, y \models \varphi \\
t, x=\varphi \mathrm{U} \psi & \text { if } \exists z \geq x .[t, z \models \psi \text { and } \forall y .(x \leq y<z) \Rightarrow t, y \models \varphi] \\
t, x=\neg a \mathrm{~S} b & \text { if } \exists z \leq x .[\lambda(z)=b \text { and } \forall y .(z<y \leq x) \Rightarrow \lambda(y) \neq a]
\end{array}
$$
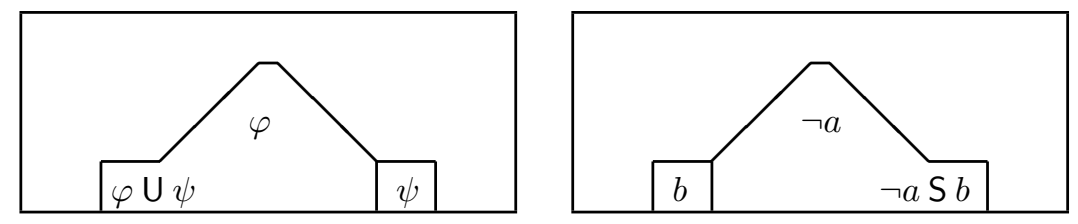

The modality $\mathrm{U}$ is the "universal" until operator defined in [3]. The modality $\mathrm{S}$ is the corresponding since operator. Note that we only use the operator $\mathrm{S}$ in the very restricted form of a fixed number of past constants.

Past modalities are essential, as indicated by the following example from [22], where the dependence relation is $a-b-c-d$. These two traces are not first-order equivalent but are bisimilar at the level of events and thus cannot be distinguished by purely future modalities.

$$
\begin{array}{rlrl}
a & \rightarrow b \rightarrow c \rightarrow b \rightarrow c \cdots & d & \rightarrow c \\
& \uparrow & \uparrow \\
d & \rightarrow c & a & \rightarrow b
\end{array}
$$

As usual, we can derive useful operators such as universal next $\operatorname{AX} \varphi=$ $\neg \mathrm{EX} \neg \varphi$, eventually in the future $\mathrm{F} \varphi=\top \mathrm{U} \varphi$ and always in the future $\mathrm{G} \varphi=$ $\neg \mathrm{F} \neg \varphi$. The modality $\mathrm{F}^{\infty} a=\mathrm{F} a \wedge \mathrm{G}(a \Rightarrow \mathrm{EXF} a)$ expresses the existence of infinitely many vertices labelled with $a$ above the current vertex.

Traces as models of formulas: We now turn our attention to defining when a trace satisfies a formula. For LTL over sequences, lifting satisfaction at positions to satisfaction by a word is quite simple: a word models a formula if its initial position models the formula. Since a trace, in general, does not have a unique 
initial position, we need to use initial formulas as introduced in [3]. These are boolean combinations of formulas EM $\varphi$, each of which asserts the existence of a minimal vertex in a trace satisfying the internal formula $\varphi$. More precisely, the set $\operatorname{LocTL}_{\Sigma}$ of initial formulas over the alphabet $\Sigma$ is defined as follows:

$$
\alpha::=\perp\left|\operatorname{EM} \varphi, \varphi \in \operatorname{LocTL}_{\Sigma}^{\mathrm{i}}\right| \neg \alpha \mid \alpha \vee \alpha
$$

The semantics of EM is given by:

$$
t=\operatorname{EM} \varphi \text { if } \exists x .(x \in \min (t) \text { and } t, x=\varphi)
$$

An initial formula $\alpha \in \operatorname{LocTL}_{\Sigma}$ defines the trace language $\mathcal{L}(\alpha)=\{t \in \mathbb{R} \mid$ $t \models \alpha\}$. We can then express various alphabetic properties using initial formulas: $\mathcal{L}(\operatorname{EM} a)=\{t \in \mathbb{R} \mid a \in \min (t)\}, \mathcal{L}(\operatorname{EMF} a)=\{t \in \mathbb{R} \mid a \in \operatorname{alph}(t)\}$, and $\mathcal{L}\left(\mathrm{EM} \mathrm{F}^{\infty} a\right)=\{t \in \mathbb{R} \mid a \in \operatorname{alphinf}(t)\}$. Therefore, for $C \subseteq \Sigma$, trace languages such as $($ alph $=C),($ alphinf $=C)$ and $(\min =C)$ are expressible in LocTL ${ }_{\Sigma}$.

The following result is immediate from the definition of $\operatorname{LocTL}_{\Sigma}$.

Proposition 2. If a trace language is expressible in $\mathrm{LocTL}_{\Sigma}$, then it is expressible in $\mathrm{FO}_{\Sigma}^{3}(<)$.

We now show that the "filtered" modalities $\mathrm{EX}_{b}$ and $\mathrm{F}_{b}$ from [10], with the following semantics, are both expressible in $\operatorname{LocTL}_{\Sigma}^{\mathrm{i}}$.

$$
\begin{aligned}
& t, x \models \mathrm{EX}_{b} \varphi \text { if } \exists y .[x \lessdot y \text { and } t, y \models \varphi \text { and } \forall z .(z \leq y \wedge \lambda(z)=b) \Rightarrow z \leq x] \\
& t, x \models \mathrm{F}_{b} \varphi \quad \text { if } \exists y .[x \leq y \text { and } t, y \models \varphi \text { and } \forall z .(z \leq y \wedge \lambda(z)=b) \Rightarrow z \leq x]
\end{aligned}
$$

Proposition 3. For any trace $t$ over some alphabet $\Sigma$, any position $x$ in $t$ and any formula $\varphi$ of $\operatorname{LocTL}_{\Sigma}^{\mathrm{i}}$

$$
t, x \models \operatorname{EX}_{b} \varphi \Longleftrightarrow t, x \models(b \wedge \operatorname{EX}(\varphi \wedge \neg b)) \vee \bigvee_{a \neq b}(a \wedge \operatorname{EX}(\varphi \wedge \neg(\neg a \mathrm{~S} b)))
$$

Let the formula $\mathrm{Safe}_{b}=(b \wedge \mathrm{AX} \neg b) \vee \bigvee_{a \neq b}(a \wedge \mathrm{AX} \neg(\neg a \mathrm{~S} b))$. Further, let $\mathrm{F}_{b}^{0} \varphi=\operatorname{Safe}_{b} \mathrm{U} \varphi$ and $\mathrm{F}_{b}^{k+1} \varphi=\operatorname{Safe}_{b} \cup \operatorname{EX}_{b}\left(\mathrm{~F}_{b}^{k} \varphi\right)$.

Proposition 4. For any trace $t \in \mathbb{R}(\Sigma, D)$, any position $x$ in $t$ and any formula $\varphi$ of $\operatorname{LocTL}_{\Sigma}^{\mathrm{i}}$

$$
t, x \models \mathrm{F}_{b} \varphi \Longleftrightarrow t, x \models \bigvee_{k \leq|\Sigma|} \mathrm{F}_{b}^{k} \varphi
$$

Now we establish some important lemmas that are critical in proving the expressive completeness of $\operatorname{LocTL}_{\Sigma}$.

Lemma 5. Let $A \subseteq \Sigma$ and $b \in \Sigma$ with $b \notin A$. For all $\varphi \in \operatorname{LocTL}_{A}^{\mathrm{i}}$, there is a formula $\widetilde{\varphi} \in \operatorname{LocTL}_{A \cup\{b\}}^{\mathrm{i}}$ such that for all $t=t_{1} b t_{2} t_{3} \in \mathbb{R}$ with $t_{1} \in \mathbb{R}$, $t_{2} \in \mathbb{R}_{A}, \min \left(t_{2}\right) \subseteq D(b)$ and $\min \left(t_{3}\right) \subseteq\{b\}$ and for all $x \in b t_{2}$ we have $b t_{2}, x \models \varphi$ iff $t, x=\widetilde{\varphi}$.

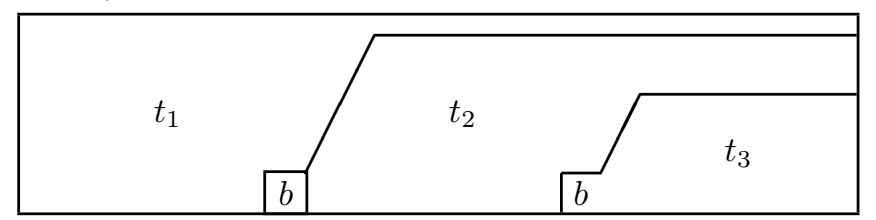


Proof Sketch. We have $\widetilde{a}=a, \widetilde{\neg \varphi}=\neg \widetilde{\varphi}, \widetilde{\varphi \vee \psi}=\widetilde{\varphi} \vee \widetilde{\psi}, \widetilde{\mathrm{EX}_{\varphi}}=\mathrm{EX}_{b} \widetilde{\varphi}, \widetilde{\varphi \mathrm{U} \psi}=$ $\mathrm{V}_{d \in A \cup\{b\}}\left((\widetilde{\varphi} \mathrm{U}(d \wedge \widetilde{\psi})) \wedge \mathrm{F}_{b}(d \wedge \widetilde{\psi})\right)$ and $\widetilde{\neg \mathrm{S} d}=(\neg c \mathrm{~S} d) \wedge \neg(\neg d \mathrm{~S} b)$.

Lemma 6. Let $A \subseteq \Sigma$ and $b \in \Sigma$ with $b \notin A$. For all $\alpha \in$ LocTL $_{A}$, there exists a formula $\bar{\alpha} \in \operatorname{LocTL}_{A \cup\{b\}}^{\mathrm{i}}$ such that for all $t=t_{1} b t_{2} t_{3} \in \mathbb{R}$ with $t_{1} \in \mathbb{R}$, $t_{2} \in \mathbb{R}_{A}$, $\min \left(t_{2}\right) \subseteq D(b)$ and $\min \left(t_{3}\right) \subseteq\{b\}$, we have $t_{2} \models \alpha$ if and only if $t, \min \left(b t_{2} t_{3}\right) \models \bar{\alpha}$.

Proof Sketch. We have $\overline{\neg \alpha}=\neg \bar{\alpha}, \overline{\alpha \vee \beta}=\bar{\alpha} \vee \bar{\beta}$ and $\overline{\operatorname{EM} \varphi}=\operatorname{EX}(\widetilde{\varphi} \wedge \neg b)$ where $\widetilde{\varphi}$ is the formula given by Lemma 5 .

Lemma 7. Let $A \subseteq \Sigma$ and $b \in \Sigma$ with $b \notin A$. For all $\alpha \in$ LocTL $_{A}$, there exists a formula $\widetilde{\alpha} \in \operatorname{LocTL}_{A \cup\{b\}}$ such that for all $t=t_{1} t_{2}$ with $t_{1} \in \mathbb{R}_{A}$ and $\min \left(t_{2}\right) \subseteq\{b\}$, we have $t_{1} \models \alpha$ if and only if $t \models \widetilde{\alpha}$.

Proof Sketch. Let $\widetilde{\varphi \vee \psi}=\widetilde{\varphi} \vee \widetilde{\psi}, \widetilde{\neg \varphi}=\neg \widetilde{\varphi}, \widetilde{\operatorname{EX} \varphi}=\operatorname{EX}(\widetilde{\varphi} \wedge \neg(\neg b S b)), \widetilde{\varphi U \psi}=$ $\widetilde{\varphi} \mathrm{U}(\widetilde{\psi} \wedge \neg(\neg b \mathrm{~S} b))$ and $\neg c \mathrm{~S} d=\neg c \mathrm{~S} d$. Then, for all $t=t_{1} t_{2}$ with $t_{1} \in \mathbb{R}_{A}$, $\min \left(t_{2}\right) \subseteq\{b\}$ and for all $x \in t_{1}$, we have $t_{1}, x \models \varphi$ if and only if $t, x \models \widetilde{\varphi}$. Finally, let $\widehat{\operatorname{EM} \varphi}=\operatorname{EM}(\widetilde{\varphi} \wedge \neg b)$.

\section{Decomposition of traces}

The proof of our main result is a case analysis based on partitioning the set of traces according to the structure of the trace. Fix a letter $b \in \Sigma$ and set $B=\Sigma \backslash\{b\}$. Using the notation introduced in Section 2, let $\Gamma^{A}=\left\{t \in \mathbb{R}_{B}^{A} \mid\right.$ $\min (t) \subseteq D(b)\}, \Gamma=\Gamma^{\emptyset}$, and $\Omega_{A}=\left\{t \in \mathbb{R}_{I(A)} \mid \min (t) \subseteq\{b\}\right\}$.

Each trace $t \in \mathbb{R}$ has a unique finite or infinite factorization $t=t_{0} b t_{1} b t_{2} \cdots$ with $t_{0} \in \mathbb{R}_{B}$ and $t_{i} \in \mathbb{R}_{B} \cap(\min \subseteq D(b))$ for all $i>0$. In particular, we have

$$
(\min =\{b\})=(b \Gamma)^{+} \uplus(b \Gamma)^{\omega} \uplus \bigcup_{\emptyset \neq A \subseteq B}(b \Gamma)^{*} b \Gamma^{A} \Omega_{A}
$$

The following two results will allow us to use this decomposition effectively in proving the expressive completeness of our logic. For this, we use $\mathrm{F}_{b}^{\infty} a=$ $\mathrm{F}_{b} a \wedge \neg \mathrm{F}_{b}\left(a \wedge \neg \mathrm{EX}_{b} \mathrm{~F}_{b} a\right)$.

Lemma 8. Let $t=t_{0} t^{\prime}$ with $t_{0}, t^{\prime} \in \mathbb{R}$ and $\min \left(t^{\prime}\right)=\{b\}$. Then,

1. $t^{\prime} \in(b \Gamma)^{\infty} \backslash\{1\}$ if and only if $t, \min \left(t^{\prime}\right) \models \beta$ with

$$
\beta=\bigvee_{C}\left(\bigwedge_{c \in C} \mathrm{~F}^{\infty} c \wedge \bigwedge_{c \notin C} \neg \mathrm{F}^{\infty} c\right)
$$

where $C$ ranges over connected subsets of $\Sigma$ such that $b \in C$ if $C \neq \emptyset$. 
2. $t^{\prime} \in(b \Gamma)^{*} b \Gamma^{A} \Omega_{A}$ if and only if $t, \min \left(t^{\prime}\right) \models \gamma$ with

$$
\gamma=\bigvee_{C \subseteq \Sigma}\left(\bigwedge_{c \notin C} \neg \mathrm{F}^{\infty} c\right) \wedge \mathrm{F}\left(b \wedge \bigwedge_{c \in C} \mathrm{~F}^{\infty} c \wedge \bigwedge_{a \in A} \mathrm{~F}_{b}^{\infty} a \wedge \bigwedge_{a \notin A} \neg \mathrm{F}_{b}^{\infty} a\right) .
$$

Note that "the" $b$ in $b \Gamma^{A} \Omega_{A}$ is characterized by the formula $b \wedge \mathrm{F}_{b}^{\infty} a$, where $a$ is any letter in $A$.

Lemma 9. Let $A \subseteq \Sigma$ and let $L \subseteq \mathbb{R}$ be a trace language recognized by a morphism $h$ from $\mathbb{M}$ into a finite monoid $S$. Then,

$$
L \cap(b \Gamma)^{*} b \Gamma^{A} \Omega_{A}=\bigcup_{\text {finite }}\left(L_{1} \cap(b \Gamma)^{*}\right) b\left(L_{2} \cap \Gamma^{A}\right)\left(L_{3} \cap \Omega_{A}\right)
$$

where the trace languages $L_{i} \subseteq \mathbb{R}$ are recognized by $h$.

\section{Expressive Completeness}

If $T$ is a finite alphabet, we define the linear temporal logic $\operatorname{LTL}_{T}(\mathrm{XU})$ by the syntax: $f::=u \in T|f \mathrm{XU} f| \neg f \mid f \vee f$.

The length of a finite or infinite word $w=w_{1} w_{2} \cdots \in T^{\infty}$ is $|w| \in \mathbb{N} \cup\{\omega\}$. For a word $w=w_{1} w_{2} \cdots \in T^{\infty}$ the semantics of $\operatorname{LTL}_{T}(\mathbf{X U})$ is given by

$$
\begin{array}{ll}
w=u & \text { if }|w|>0 \text { and } w_{1}=u \\
w=f \times \mathbf{X U} g \text { if } \quad \exists j \in \mathbb{N} \text { with } 1<j \leq|w|+1 \text { and } \\
\\
w_{j} w_{j+1} \cdots \models g \text { and } w_{k} w_{k+1} \cdots \models f, \forall 1<k<j .
\end{array}
$$

Note that if $w=f \mathbf{X U} g$ then $w$ is nonempty. A formula $f \in \mathrm{LTL}_{T}(\mathrm{XU})$ defines the word language $\mathcal{L}(f)=\left\{w \in T^{\infty}|w|=f\right\}$. We use the following proposition which is a consequence of several results on the equivalence between aperiodic word languages, star-free word languages, first order word languages and word languages expressible in $\mathrm{LTL}_{T}(\mathrm{XU})[18,12,14,20,8,15,16,2]$.

Proposition 10. Every aperiodic word language $K \in T^{\infty}$ is expressible in $\operatorname{LTL}_{T}(\mathrm{XU})$.

We fix $T=h(b \Gamma)$ and we define the mapping $\sigma:(b \Gamma)^{\infty} \rightarrow T^{\infty}$ by $\sigma(t)=$ $h\left(b t_{1}\right) h\left(b t_{2}\right) \cdots$ if $t=b t_{1} b t_{2} \cdots$ with $t_{i} \in \Gamma$ for $i \geq 1$. Note that the mapping $\sigma$ is well-defined since each trace $t \in(b \Gamma)^{\infty}$ has a unique factorization $t=b t_{1} b t_{2} \cdots$ with $t_{i} \in \Gamma$ for $i \geq 1$.

Lemma 11. Let $L \subseteq \mathbb{R}$ be recognized by $h$. Then,

1. $L \cap(b \Gamma)^{\omega}=\sigma^{-1}(K)$ for some $K$ expressible in $\mathrm{LTL}_{T}(\mathrm{XU})$.

2. $L \cap(b \Gamma)^{+}=\sigma^{-1}(K)$ for some $K$ expressible in $\mathrm{LTL}_{T}(\mathrm{XU})$.

Next we show show how to lift an $\operatorname{LTL}_{T}(\mathrm{XU})$ formula for $K \subseteq T^{\infty}$ to a LocTL ${ }^{\mathrm{i}}$ formula for $\sigma^{-1}(K) \in(b \Gamma)^{\infty}$.

Lemma 12. Suppose that any aperiodic trace language over $B$ is expressible in $\operatorname{LocTL}_{B}$. Then, for all $f \in \mathrm{LTL}_{T}(\mathrm{XU})$ there exists $\widetilde{f} \in \operatorname{LocTL}_{\Sigma}^{\mathrm{i}}$ such that for all $t=t_{1} t^{\prime}$ with $t_{1} \in \mathbb{R}$ and $t^{\prime} \in(b \Gamma)^{\infty} \backslash\{1\}$, we have $\sigma\left(t^{\prime}\right) \models f$ iff $t, \min \left(t^{\prime}\right) \models \widetilde{f}$. 
Proof Sketch. The formula $\widetilde{f}$ is defined by structural induction. We let $\widetilde{f \vee g}=$ $\widetilde{f} \vee \widetilde{g}, \widetilde{\neg f}=\neg \widetilde{f}, \widetilde{f \times U} g=\operatorname{EX}((\neg b \vee \widetilde{f}) \cup(b \wedge \widetilde{g}))$. The difficult case is when $f=$ $s \in T$. For all $r \in S$, the trace language $h^{-1}(r) \cap \mathbb{M}_{B}$ is aperiodic and therefore expressible in $\operatorname{LocTL}_{B}$ by the hypothesis of the lemma: we find $\alpha_{r} \in \operatorname{LocTL}_{B}$ such that for all $t^{\prime \prime} \in \mathbb{M}_{B}, h\left(t^{\prime \prime}\right)=r$ if and only if $t^{\prime \prime} \models \alpha_{r}$. Let $\overline{\alpha_{r}} \in \operatorname{LocTL}_{\Sigma}^{\mathrm{i}}$ be the formula obtained using Lemma 6 . We let $\widetilde{s}=\bigvee_{h(b) \cdot r=s} \overline{\alpha_{r}}$.

Lemma 13. Suppose that any aperiodic trace language over $B$ is expressible in $\operatorname{LocTL}_{B}$. Let $A \subseteq \Sigma$ be non-empty and let $f \in \mathrm{LTL}_{T}(\mathrm{XU})$. There exists $\tilde{f} \in \operatorname{LocTL}_{\Sigma}^{\mathrm{i}}$ such that for all $t=t_{1} t_{2} t_{3}$ with $t_{1} \in \mathbb{R}, t_{2} \in(b \Gamma)^{*}, t_{3} \in b \Gamma^{A} \Omega_{A}$, we have $\sigma\left(t_{2}\right) \models f$ iff $t, \min \left(t_{2} t_{3}\right) \models \widetilde{f}$.

Lemma 14. Suppose that for any proper subset $A$ of $\Sigma$, any aperiodic trace language over $A$ is expressible in $\operatorname{LocTL}_{A}$. Let $L \subseteq \mathbb{R}$ be an aperiodic trace language over $\Sigma$. Then, for all $b \in \Sigma$, there exists $\varphi \in \operatorname{LocTL}_{\Sigma}^{\mathrm{i}}$ such that for all $t=t_{0} t^{\prime}$ with $t_{0}, t^{\prime} \in \mathbb{R}$ and $\min \left(t^{\prime}\right)=\{b\}, t^{\prime} \in L$ iff $t, \min \left(t^{\prime}\right) \models \varphi$.

Proof. We prove this lemma by induction on the size of the alphabet $\Sigma$. If $\Sigma=\emptyset$ then there is nothing to prove. Now, suppose that $\Sigma \neq \emptyset$ and let $b \in \Sigma$. We assume that $L$ is recognized by the aperiodic morphism $h: \mathbb{M} \rightarrow S$. Now, $L \cap(\min =\{b\})$ can be written as

$$
\left(L \cap\left((b \Gamma)^{\infty} \backslash\{1\}\right)\right) \uplus \bigcup_{\emptyset \neq A \subseteq B}\left(L \cap(b \Gamma)^{*} b \Gamma^{A} \Omega_{A}\right) .
$$

By Lemma 11 we get $L \cap\left((b \Gamma)^{\infty} \backslash\{1\}\right)=\sigma^{-1}(\mathcal{L}(f))$ for some $f \in \operatorname{LTL}_{T}(\mathrm{XU})$. From the hypothesis, aperiodic languages over $B$ are expressible in $\operatorname{LocTL}_{B}$. Hence, we can apply Lemma 12 and we get $\widetilde{f}$ such that for all $t=t_{0} t^{\prime}$ with $t_{0} \in \mathbb{R}$ and $t^{\prime} \in(b \Gamma)^{\infty} \backslash\{1\}$, we have $\sigma\left(t^{\prime}\right) \models f$ iff $t, \min \left(t^{\prime}\right) \models \widetilde{f}$. We conclude this case taking $\varphi=\beta \wedge \widetilde{f}$ where $\beta$ is defined in Lemma 8 .

Now, we consider $L \cap(b \Gamma)^{*} b \Gamma^{A} \Omega_{A}$ where $\emptyset \neq A \subseteq B$. By Lemma 9,

$$
L \cap(b \Gamma)^{*} b \Gamma^{A} \Omega_{A}=\bigcup_{\text {finite }}\left(L_{1} \cap(b \Gamma)^{*}\right) b\left(L_{2} \cap \Gamma^{A}\right)\left(L_{3} \cap \Omega_{A}\right)
$$

where each $L_{i}$ is an aperiodic language recognized by $h$. Thus, it suffices to show that for aperiodic languages $L_{1}, L_{2}$ and $L_{3}$ recognized by $h$, there is a formula $\varphi$ such that for all $t=t_{0} t^{\prime}$ with $t_{0}, t^{\prime} \in \mathbb{R}$ and $\min \left(t^{\prime}\right)=\{b\}$, we have $t, \min \left(t^{\prime}\right) \models \varphi$ if and only if $t^{\prime} \in\left(L_{1} \cap(b \Gamma)^{*}\right) b\left(L_{2} \cap \Gamma^{A}\right)\left(L_{3} \cap \Omega_{A}\right)$.

By Lemma 11 we get $L_{1} \cap(b \Gamma)^{*}=\sigma^{-1}\left(\mathcal{L}\left(f_{1}\right)\right)$ for some $f_{1} \in \operatorname{LTL}_{T}(\mathrm{XU})$. From the hypothesis, aperiodic languages over $B$ are expressible in $\operatorname{LocTL}_{B}$. Hence, we can apply Lemma 13 and we get $\widetilde{f}_{1}$ such that for all $t=t_{0} t_{1} t^{\prime \prime}$ with $t_{0} \in \mathbb{R}$ and $t_{1} \in(b \Gamma)^{*}$, and $t^{\prime \prime} \in b \Gamma^{A} \Omega_{A}$, we have $t_{1} \in L_{1}$ iff $t, \min \left(t_{1} t^{\prime \prime}\right) \models \widetilde{f}_{1}$.

Using again the hypothesis of the lemma, we get some formula $\alpha_{2} \in$ LocTL $_{B}$ such that $L_{2} \cap \mathbb{R}_{B}=\mathcal{L}\left(\alpha_{2}\right)$. By Lemma 6 we find $\overline{\alpha_{2}} \in \operatorname{LocTL}_{\Sigma}^{\mathrm{i}}$ such that for all $t=t_{0} t_{1} b t_{2} t_{3}$ with $t_{0} \in \mathbb{R}$ and $t_{1} \in(b \Gamma)^{*}, t_{2} \in \Gamma^{A}$ and $t_{3} \in \Omega_{A}$, we have $t_{2} \in L_{2}$ iff $t, \min \left(b t_{2} t_{3}\right) \models \overline{\alpha_{2}}$. 
Finally, $L_{3}$ is an aperiodic trace language over a smaller alphabet (since $A \neq \emptyset, I(A)$ is a proper subset of $\Sigma$ ) and hence by induction hypothesis there is a formula $\varphi_{3}$ such that for all $t=t_{0} t_{1} b t_{2} t_{3}$ with $t_{0} \in \mathbb{R}$ and $t_{1} \in(b \Gamma)^{*}, t_{2} \in \Gamma^{A}$ and $t_{3} \in \Omega_{A}$ with $t_{3} \neq 1$, we have $t_{3} \in L_{3}$ iff $t, \min \left(t_{3}\right) \models \varphi_{3}$.

Putting these three pieces together we let

$$
\psi=\tilde{f}_{1} \wedge \mathrm{F}\left(b \wedge \mathrm{F}_{b}^{\infty} a \wedge \overline{\alpha_{2}} \wedge\left(\varphi_{4} \vee \mathrm{F}_{b} \operatorname{EX}\left(b \wedge \varphi_{3}\right)\right)\right)
$$

with $\varphi_{4}=\perp$ if $1 \notin L_{3}$ and $\varphi_{4}=\neg \mathrm{EXF} b$ otherwise. Then, for all $t=t_{0} t_{1} b t_{2} t_{3}$ with $t_{0} \in \mathbb{R}$ and $t_{1} \in(b \Gamma)^{*}, t_{2} \in \Gamma^{A}$ and $t_{3} \in \Omega_{A}$, we get from the above discussion that $t_{1} b t_{2} t_{3} \in L_{1} b L_{2} L_{3}$ if and only if $t, \min \left(t_{1} b t_{2} t_{3}\right) \models \psi$. We complete the proof with $\varphi=\gamma \wedge \psi$ where $\gamma$ is the formula defined in Lemma 8.

Theorem 15. Any aperiodic real trace language over $\mathbb{R}(\Sigma, D)$ is expressible in $\operatorname{LocTL}_{\Sigma}$.

Proof. The proof proceeds by induction on the size of $\Sigma$. When $\Sigma=\{a\}$ is a singleton, $L$ is either a finite set or the union of a finite set and a set of the form $a^{n} a^{*}$ for some $n \geq 0$. In both cases, it is easy to check that $L$ is expressible in $\operatorname{LocTL}_{\Sigma}$.

For the inductive step, assume that the theorem holds for any aperiodic language over any proper subset of $\Sigma$. Let $L$ be recognized by an aperiodic morphism $h: \mathbb{M} \rightarrow S$. Let $b \in \Sigma$ and $B=\Sigma \backslash\{b\}$ as usual. We can show as in Lemma 9 that $L$ can be written as follows:

$$
L=\bigcup_{\text {finite }}\left(L_{1} \cap \mathbb{R}_{B}\right)\left(L_{2} \cap(\min \subseteq\{b\})\right)
$$

where $L_{1}$ and $L_{2}$ are languages recognized by the same aperiodic morphism $h$.

Since the decomposition of any trace $t \in \mathbb{R}$ as $t_{1} t_{2}$ with $t_{1} \in \mathbb{R}_{B}$ and $t_{2} \in$ $(\min \subseteq\{b\})$ is unique, the above decomposition can be rewritten as

$$
L=\bigcup_{\text {finite }}\left(L_{1} \cap \mathbb{R}_{B}\right)(\min \subseteq\{b\}) \cap\left(\mathbb{R}_{B}\left(L_{2} \cap(\min \subseteq\{b\})\right)\right)
$$

Now, by the induction hypothesis, there is formula $\alpha_{1}$ in $\operatorname{LocTL}_{B}$ such that for $t_{1} \in \mathbb{R}_{B}, t_{1} \models \alpha_{1}$ if and only if $t_{1} \in L_{1}$. Thus, by Lemma 7 , there is a formula $\widetilde{\alpha_{1}}$ in $\operatorname{LocTL}_{\Sigma}$ such that $t \models \widetilde{\alpha_{1}}$ if and only if $t_{1} \models \alpha_{1}$ whenever $t=t_{1} t_{2}$ with $t_{1} \in \mathbb{R}_{B}$ and $\min \left(t_{2}\right) \subseteq\{b\}$. Thus, $\left(L_{1} \cap \mathbb{R}_{B}\right)(\min \subseteq\{b\})=\mathcal{L}\left(\widetilde{\alpha_{1}}\right)$.

Since we have assumed expressive completeness for every proper subset of $\Sigma$, by Lemma 14 there is a formula $\varphi_{2}$ such that for any $t=t_{1} t_{2}$ with $\min \left(t_{2}\right)=b$, $t_{2} \in L_{2}$ if and only if $t, \min \left(t_{2}\right)=\varphi_{2}$. Consider the formula $\alpha=\alpha^{\prime} \vee \operatorname{EM}((b \wedge$ $\left.\left.\varphi_{2}\right) \vee\left(\neg b \wedge \mathrm{F}_{b} \operatorname{EX}\left(b \wedge \varphi_{2}\right)\right)\right)$ where $\alpha^{\prime}=\perp$ if $1 \notin L_{2}$ and $\alpha^{\prime}=\neg \mathrm{EMF} b$ otherwise. Then, $t \models \alpha$ if and only if either $t \in \mathbb{R}_{B}$ and $1 \in L_{2}$, or there is a minimal $b$ event $x$ in the trace $t$ and $t, x \models \varphi_{2}$. That is $t=t_{1} t_{2}$ with $t_{1} \in \mathbb{R}_{B}, \min \left(t_{2}\right)=\{b\}$ and $t_{2} \in L_{2}$. Thus $\mathbb{R}_{B}\left(L_{2} \cap(\min \subseteq\{b\})\right)=\mathcal{L}(\alpha)$ is also expressible in LocTL ${ }_{\Sigma}$. 


\section{References}

1. B. Adsul and M. Sohoni. Complete and tractable local linear time temporal logics over traces. In Proc. of ICALP'02, LNCS 2380, 926-937. Springer Verlag, 2002.

2. J. Cohen, D. Perrin, and J.-E. Pin. On the expressive power of temporal logic. Journal of Computer and System Sciences, 46:271-295, 1993.

3. V. Diekert and P. Gastin. Local temporal logic is expressively complete for cograph dependence alphabets. In Proc. of LPAR'01, LNAI 2250, 55-69. Springer Verlag, 2001.

4. V. Diekert and P. Gastin. LTL is expressively complete for Mazurkiewicz traces. Journal of Computer and System Sciences, 64:396-418, 2002.

5. V. Diekert and G. Rozenberg, editors. The Book of Traces. World Scientific, Singapore, 1995.

6. W. Ebinger. Charakterisierung von Sprachklassen unendlicher Spuren durch Logiken. Dissertation, Institut für Informatik, Universität Stuttgart, 1994.

7. W. Ebinger and A. Muscholl. Logical definability on infinite traces. Theoretical Computer Science, 154:67-84, 1996.

8. D. Gabbay, A. Pnueli, S. Shelah, and J. Stavi. On the temporal analysis of fairness. In Proc. of PoPL'80, 163-173, Las Vegas, Nev., 1980.

9. P. Gastin and D. Kuske. Satisfiability and model checking for MSO-definable temporal logics are in PSPACE. To appear in Proc. of CONCUR'03.

10. P. Gastin and M. Mukund. An elementary expressively complete temporal logic for Mazurkiewicz traces. In Proc. of ICALP'02, LNCS 2380, 938-949. Springer Verlag, 2002

11. P. Gastin, M. Mukund, and K. Narayan Kumar. Local LTL with past constants is expressively complete for Mazurkiewicz traces. Tech. Rep. 2003-008, LIAFA, Université Paris 7 (France), 2003.

12. J.A.W. Kamp. Tense Logic and the Theory of Linear Order. PhD thesis, University of California, Los Angeles, California, 1968.

13. A. Mazurkiewicz. Concurrent program schemes and their interpretations. DAIMI Rep. PB 78, Aarhus University, Aarhus, 1977.

14. R. McNaughton and S. Papert. Counter-Free Automata. MIT Press, 1971.

15. D. Perrin. Recent results on automata and infinite words. In Proc. of MFCS'84, LNCS 176, 134-148. Springer Verlag, 1984.

16. D. Perrin and J.-E. Pin. First order logic and star-free sets. Journal of Computer and System Sciences, 32:393-406, 1986.

17. A. Pnueli. The temporal logic of programs. In FOCS'r7, 46-57, 1977.

18. M.-P. Schützenberger. On finite monoids having only trivial subgroups. Information and Control, 8:190-194, 1965.

19. P.S. Thiagarajan and I. Walukiewicz. An expressively complete linear time temporal logic for Mazurkiewicz traces. In Proc. of LICS'97, 183-194, 1997.

20. W. Thomas. Star-free regular sets of $\omega$-sequences. Information and Control, 42:148-156, 1979.

21. I. Walukiewicz. Difficult configurations - on the complexity of LTrL. In Proc. of ICALP'98, LNCS 1443, 140-151. Springer Verlag, 1998.

22. I. Walukiewicz. Local logics for traces. Journal of Automata, Languages and Combinatorics, 7(2):259-290, 2002.

23. Th. Wilke. Classifying discrete temporal properties. In Proc. of STACS'99, LNCS 1563, 32-46. Springer Verlag, 1999.

24. W. Zielonka. Notes on finite asynchronous automata. R.A.I.R.O. - Informatique Théorique et Applications, 21:99-135, 1987. 